\title{
Plasma appearance and correlation between coffee and green tea metabolites in human subjects
}

\author{
Mathieu Renouf*, Philippe Guy, Cynthia Marmet, Karin Longet, Anne-Lise Fraering, Julie Moulin, \\ Denis Barron, Fabiola Dionisi, Christophe Cavin, Heike Steiling and Gary Williamson \\ Nestlé Research Center, Nestec Limited, Vers-Chez-Les-Blanc, 1000 Lausanne 26, Switzerland
}

(Received 16 March 2010 - Revised 25 May 2010 - Accepted 2 June 2010 - First published online 9 August 2010)

Coffee and green tea are two of the most widely consumed hot beverages in the world. Their respective bioavailability has been studied separately, but absorption of their respective bioactive phenolics has not been compared. In a randomised cross-over design, nine healthy subjects drank instant coffee and green tea. Blood samples were collected over $12 \mathrm{~h}$ and at $24 \mathrm{~h}$ to assess return to baseline. After green tea consumption, (-)-epigallocatechin (EGC) was the major catechin, appearing rapidly in the plasma; (-)-EGC gallate (EGCg) and (-)-epicatechin (EC) were also present, but (-)-EC gallate and $\mathrm{C}$ were not detected. Dihydroferulic acid and dihydrocaffeic acid were the major metabolites that appeared after coffee consumption with a long time needed to reach maximum plasma concentration, suggesting metabolism and absorption in the colon. Other phenolic acid equivalents (caffeic acid (CA), ferulic acid (FA) and isoferulic acid (iFA)) were detected earlier, and they peaked at lower concentrations. Summations of the plasma area under the curves (AUC) for the measured metabolites showed 1.7-fold more coffee-derived phenolic acids than green tea-derived catechins $(P=0 \cdot 0014)$. Furthermore, we found a significant correlation between coffee metabolites based on AUC. Inter-individual differences were observed, but individuals with a high level of CA also showed a correspondingly high level of FA. However, no such correlation was observed between the tea catechins and coffee phenolic acids. Correlation between AUC and maximum plasma concentration was also significant for CA, FA and iFA and for EGCg. This implies that the mechanisms of absorption for these two classes of compounds are different, and that a high absorber of phenolic acids is not necessarily a high absorber of catechins.

Coffee: Green tea: Bioavailability: Phenolic acids: Catechins

Coffee contains phenolic compounds called hydroxycinnamates, which principally consist of chlorogenic acids, a family of trans-cinnamicacidsconjugated withquinicacid. The main chlorogenic acid in coffee is 5-caffeoylquinic acid ${ }^{(1)}$. Tea is an infusion of the leaves of the Camellia sinensis plant, and is the most widely consumed beverage in the world after water ${ }^{(2)}$. Some biologically active components of tea include flavonoids, caffeine and fluoride. Both of these caffeine-containing beverages are greatly consumed around the world, and because they contain many different bioactives, including polyphenols (Polyphenols. In the present study, phenolic acid equivalents derived from coffee chlorogenic acids and catechins derived from green tea are the specific compounds described as coffee and green tea polyphenols, respectively), they are studied for their potential healthpromoting effects ${ }^{(3-5)}$. Even in Japan, well known for green tea consumption, the contribution of polyphenols in coffee to the Japanese diet is at least equal to that of green tea $^{(6)}$. However, the bioavailability (Bioavailability. In the present study, bioavailability is limited to plasma appearance of polyphenols over time) and metabolism of these bioactives remain to be fully investigated, especially for coffee. Indeed, only a few papers have investigated how chlorogenic and phenolic acids present in coffee are absorbed in the plasma and/or excreted in the urine so $\operatorname{far}^{(7-11)}$. Bioavailability of the phenolic acids present in coffee is difficult to fully quantify, since it contains many components that cannot be clearly identified (e.g. melanoidin metabolites) due to the lack of proper standards. Because the bioavailability of green tea catechins appears to be more simple, studies on catechin bioavailability seem to provide clearer answers ${ }^{(12-21)}$.

In the present study, we investigated the bioavailability of coffee chlorogenic acids expressed as phenolic acid equivalents and the bioavailability of green tea catechins in the same volunteers in a cross-over design. To make the comparison feasible, five major metabolites were studied for each beverage, and plasma appearance of these metabolites was summed and expressed in molar concentrations to facilitate the assessment and comparison of the two different classes of bioactives. We also investigated if there was any

Abbreviations: AUC, area under the curve; $C_{\max }$, maximum plasma concentration; CA, caffeic acid; FA, ferulic acid; iFA, isoferulic acid; DHCA, dihydrocaffeic acid; DHFA, dihydroferulic acid; EC, (-)-epicatechin; EGC, (-)-epigallocatechin; EGCg, (-)-EGC gallate; $T_{\max }$, time needed to reach maximum plasma concentration.

* Corresponding author: Dr M. Renouf, fax +41 2178594 86, email mathieu.renouf@rdls.nestle.com 
correlation between the metabolites. Using bioavailability parameters, such as area under the curve (AUC) and maximum plasma concentration $\left(C_{\max }\right)$, we also tested the hypothesis that an apparent high absorber of coffee phenolics could also be an apparent high absorber of tea catechins.

\section{Materials and methods}

\section{Subjects}

Twelve healthy subjects were recruited for the present study. Nine subjects (four males and five females) completed the study. The study was approved by the Ethics Committee of Clinical Research of the University of Lausanne, Switzerland (protocol reference 136/07). Inclusion criteria included age 18-50 years; healthy, average and regular coffee consumption of $1-5 \mathrm{cups} / \mathrm{d}$; and non-smoking.

\section{Study design}

The original protocol was a controlled four-treatment crossover study. Two of the treatments were considered for secondary objectives, and will not be discussed in the present paper. At 1 week before the first treatment, BMI was measured. For $24 \mathrm{~h}$ before each treatment until the end of the sampling period, the ingestion of coffee, tea, cola, alcohol, whole-grain cereal (white bread allowed) or any medication was not allowed. Only water could be drunk during the night and in the morning before the treatment. On each study day, the subjects arrived fasted early in the morning at the metabolic unit. Baseline blood was sampled, and then one of the treatments was given to the subjects. Treatments included $4 \mathrm{~g}$ of soluble instant coffee dissolved in $400 \mathrm{ml}$ of water (coffee treatment) or $400 \mathrm{ml}$ of $1.25 \%$ green tea infusion (green tea treatment). The composition of these beverages is given in Table 1, showing that the amounts of polyphenols in coffee and tea given to the subjects were very similar. Each subject was given the 'coffee' or the 'green tea' treatment once at random in a cross-over fashion. The washout period between the treatments was at least 1 week. On each study day, blood was collected at $0.5,1,1.5,2,3,4,5,6,8$, 10,11 and $12 \mathrm{~h}$ after one of the treatments. A standard lunch and dinner were provided at the metabolic unit. The subjects were encouraged to drink as much as they

Table 1. Composition of polyphenols present in the coffee and green tea given to the subjects

\begin{tabular}{lrlr}
\hline Coffee $^{*}$ & $\mathrm{mg}$ & Green teat & $\mathrm{mg}$ \\
\hline CQA & 256 & C & 7 \\
FQA & 37 & EC & 37 \\
di-CQA & 42 & ECg & 39 \\
& & EGC & 81 \\
Total & 335 & EGCg & 134 \\
& & Total & 298 \\
\hline
\end{tabular}

CQA, caffeoylquinic acid; FQA, feruloylquinic acid; di-CQA, di-caffeoylquinic acid EC, (-)-epicatechin; ECG, (-)-epicatechin gallate; EGC, (-)-epigallocatechin; EGCg, (-)-epigallocatechin gallate.

${ }^{*}$ Coffee was prepared using $4 \mathrm{~g}$ of soluble coffee dissolved in $400 \mathrm{ml}$ of hot water.

† Green tea was prepared using a $1.25 \%$ infusion stirred for $3 \mathrm{~min}$ in $400 \mathrm{ml}$ of hot water. wanted, and water was available ad libitum. At $24 \mathrm{~h}$ after the treatment, a final blood sample was collected to assess return to baseline.

\section{Plasma extraction after coffee consumption and analyses via LC-electronspray ionisation-MS/MS}

The method that was used to extract phenolic acids from plasma samples was performed exactly as described in detail elsewhere, and validated using authentic synthetic standards of phenolic acid aglycones, sulphates and glucuronides spiked into blank plasma ${ }^{(11,22)}$. The method that was used to quantify coffee phenolics in the plasma has been described in detail elsewhere ${ }^{(11,22)}$.

\section{Plasma extraction after green tea consumption and analyses via HPLC with electrochemical detection}

Extraction of plasma samples after ingestion of green tea was done via a liquid-liquid extraction on ice. In summary, $12 \mu \mathrm{l}$ of a solution containing $10 \%$ vitamin $\mathrm{C}$ and $1 \%$ EDTA (to stabilise), $20 \mu \mathrm{l}$ of $50 \mathrm{mmol} / 1 \mathrm{~K}_{3} \mathrm{PO}_{4}$ (pH 7.4, to achieve the optimal $\mathrm{pH}$ for enzymatic activity), $10 \mu \mathrm{l}$ of internal standard (ethyl gallate, final concentration $=1 \mu \mathrm{mol} / \mathrm{l}$ ), $200 \mu \mathrm{l}$ of plasma and $100 \mu \mathrm{l}$ of enzymatic mix (500units $\beta$-glucuronidase +10 units sulphatase in $0.1 \mathrm{~mol} / \mathrm{l}$ sodium acetate, $\mathrm{pH} 5.5$ ) were mixed in $1.5 \mathrm{ml}$ amber Eppendorf tubes. The tubes were closed, vortexed for $5 \mathrm{~s}$, incubated at $37^{\circ} \mathrm{C}$ for $30 \mathrm{~min}(600 \mathrm{rpm})$ and then immediately put on ice. Extraction was done with $1 \mathrm{ml}$ of ethyl acetate, vortexing was done for $30 \mathrm{~s}$ and centrifugation was done for $5 \mathrm{~min}$ at $4^{\circ} \mathrm{C}$ for $15000 \mathrm{rpm}$. The supernatant was transferred into a new $2 \mathrm{ml}$ amber Eppendorf vial (on ice), and $10 \mu \mathrm{l}$ of a solution containing $0.5 \%$ vitamin $\mathrm{C}$ and $0.05 \%$ EDTA were added. The lower phase was extracted again with ethyl acetate, vortexed for $30 \mathrm{~s}$ and centrifuged for $5 \mathrm{~min}$ at $4^{\circ} \mathrm{C}$ for $15000 \mathrm{rpm}$. The supernatant that was obtained was combined with the previous one, and $10 \mu \mathrm{l}$ of a solution with $0.5 \%$ vitamin $\mathrm{C}$ and $0.05 \%$ EDTA were added to stabilise the phase. The latter was dried under $\mathrm{N}_{2}$ in a cold environment. The residue was resuspended in $200 \mu \mathrm{l}$ of $20 \%$ methanol in water, vortexed for $10 \mathrm{~s}$ and centrifuged for $5 \mathrm{~min}$ at $4^{\circ} \mathrm{C}$ for $15000 \mathrm{rpm}$. A quantity of $100 \mu \mathrm{l}$ of the sample was transferred into an amber HPLC vial for injection, and the remainder was kept at $-80^{\circ} \mathrm{C}$ for back-up analyses.

The method that was used to quantify catechins in the plasma was performed using HPLC coupled with electrochemical detection (Coularray ${ }^{\circledR}$ detector model 5600A fitted with solvent delivery module 582, GuardStat (Euroservice, Genova, Italy)). HPLC analyses were performed on a Macherey-Nagel Nucleosil C18 $5 \mu \mathrm{m} 250 \times 3 \mathrm{~mm}$ column with a Precolumn Macherey-Nagel CC 8/3 Nucleosil 100-5 C18 (Oensingen, Switzerland). The mobile phases consisted of phase A: $75 \mathrm{mmol}$ citric acid and $25 \mathrm{mmol}$ ammonium acetate, $\mathrm{pH} 2.66$, and phase $\mathrm{B}: 50 \%$ phase $\mathrm{A}$ and $50 \%$ acetonitrile . The gradient programme was as follows: $0 \mathrm{~min}, 75 \% \mathrm{~A}$; $0-13 \mathrm{~min}, 75 \% \mathrm{~A} ; 13-22 \mathrm{~min}, 70 \% \mathrm{~A} ; 22-35 \mathrm{~min}, 50 \%$ A; $35-42 \mathrm{~min}, 0 \% \mathrm{~A}$; and $42-50 \mathrm{~min}, 97 \% \mathrm{~A}$. The flow rate was $0.5 \mathrm{ml} / \mathrm{min}$, and the injection volume was $50 \mu \mathrm{l}$. The detection was performed with cell potentials set at $-90,-10,70,150,230,310,400$ and $480 \mathrm{mV}$. To quantify 
detectable catechins present in the samples, a standard curve was constructed using known spiked samples, and the ratio of the sum of the peak heights of the substance to that of the internal standard was plotted $v$. the concentration. Similar ratios obtained from unknown plasma samples generated in the present study were then quantified based on this standard curve. For each patient, a standard curve was constructed from his/her baseline plasma spiked with known concentration of catechins to avoid inter-individual variability and plasma matrix effects.

\section{Statistical analyses}

Participants were randomised to sequences issued from a $4 \times 4$ Williams Latin square.

The areas under the available plasma curve of coffee phenolic acids were calculated over $12 \mathrm{~h}$ duration using the trapezoidal method.

All the calculated parameters (AUC, $C_{\max }$ and time needed to reach maximum plasma concentration $\left(T_{\max }\right)$ ) were skewed to the right, and they needed a $\log$ transformation to reach normality. Thus, data are described by the geometric mean and the first and third quartiles.

A linear mixed model was used for the log-transformed data with treatment as the fixed effect and volunteers as the random effect. The estimations of the treatment effects are presented as the ratio of geometric means and their $95 \%$ CI. Correlations between metabolites based on AUC and $C_{\max }$ as well as the $\mathrm{AUC} / C_{\max }$ correlation for each individual metabolite were performed using regression analyses with significance of correlation set at $P<0 \cdot 05$.

Statistical analyses were done with Statistical Analysis Systems software (version 9.1; SAS Institute, Cary, NC, USA). The rejection level in the statistical tests is equal to $5 \%$.

\section{Results}

\section{Plasma pharmacokinetics of coffee}

Some data on the bioavailability of coffee obtained from the present study have been already described elsewhere ${ }^{(11)}$. In summary, we found that caffeic acid (CA), ferulic acid (FA) and isoferulic acid (iFA) equivalents peaked early ( $T_{\max }$ at approximately $1 \mathrm{~h}$ ), suggesting absorption in the small intestine. On the other hand, dihydroferulic acid (DHFA) and dihydrocaffeic acid (DHCA) appeared in the plasma later $\left(T_{\max }\right.$ at approximately $8-12 \mathrm{~h}$ ), suggesting metabolism and absorption in the colon. Green tea metabolites were not detected in the plasma samples of subjects who drank coffee. Calculation of individual AUC was done for each subject and each metabolite with the data available between 0 and $12 \mathrm{~h}$, with the $24 \mathrm{~h}$ time point being used only to assess return to baseline. Large inter-individual variability was observed, especially for the colonic metabolites (Fig. 1).

\section{Plasma pharmacokinetics of green tea}

We established a method to measure C, (- )-epicatechin (EC), (-)-EC gallate, (-)-epigallocatechin (EGC) and (-)-EGC gallate $(\mathrm{EGCg})$ in the plasma after green tea consumption. However, under the in vivo conditions in the present human

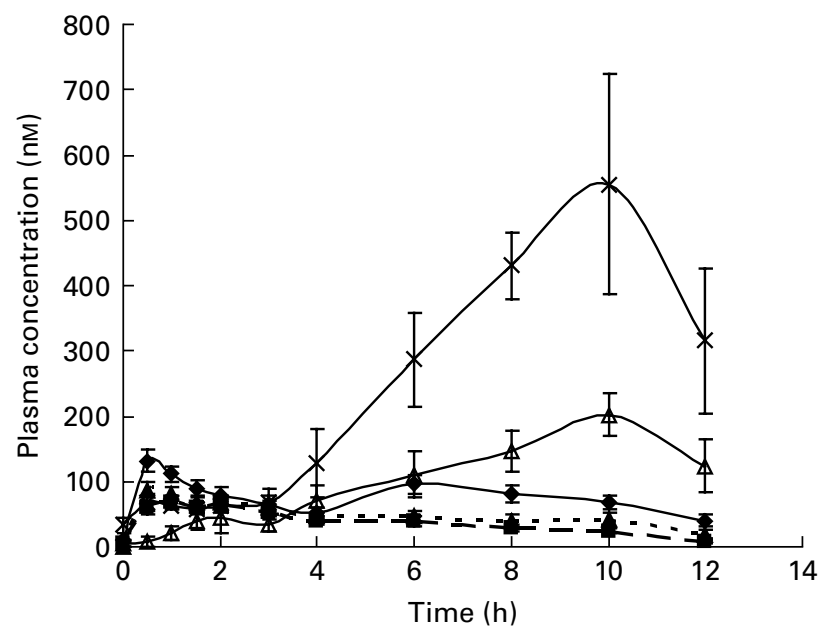

Fig. 1. Mean plasma concentration of caffeic acid (-口-) equivalents, ferulic acid $(-\bullet-)$ equivalents, isoferulic acid $(--\boldsymbol{\Delta}--)$ equivalents, dihydroferulic acid $(-\times-)$ and dihydrocaffeic acid $(-\triangle-)$ in healthy volunteers after ingestion of coffee. Values are geometric means and $95 \% \mathrm{Cl}(n 9)$.

intervention study, only EC, EGC and EGCg could be detected. Coffee metabolites were not detected in the plasma samples of subjects who drank green tea. These compounds were absorbed very quickly with a $C_{\max }$ about $1-2 \mathrm{~h}$ after ingestion. Clearance from plasma was also rapid, and was back to baseline $6-8 \mathrm{~h}$ after ingestion with a monophasic response (Fig. 2).

Comparison of plasma pharmacokinetics of coffee v. green tea

The comparison was done by summing the individual $\mathrm{AUC}_{0-12 \mathrm{~h}}$ of CA, FA, iFA, DHFA and DHCA for coffee and the individual $\mathrm{AUC}_{0-12 \mathrm{~h}}$ of $\mathrm{EC}, \mathrm{EGC}$ and $\mathrm{EGCg}$ for green tea. The bioavailability of the compounds calculated over the $12 \mathrm{~h}$ was significantly higher in coffee than in green tea (mean ratio of coffee:green tea $=1.72 ; 95 \% \mathrm{CI}$ $1 \cdot 27,2 \cdot 34 ; P=0.0014$ ) (Fig. 3). This comparison was possible due to the fact that the amounts of coffee and tea polyphenols given to the subjects were similar (Table 1).

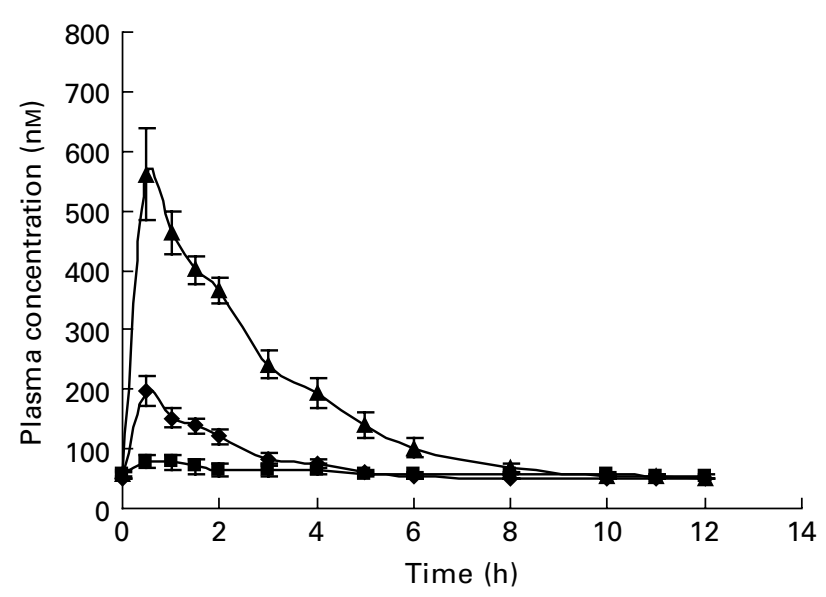

Fig. 2. Mean plasma concentration of $(-)$-epicatechin $(-\bullet-),(-)$-epigallocatechin gallate $(-\mathbf{\square}-)$ and $(-)$-epigallocatechin $(-\boldsymbol{\Delta}-)$ in healthy volunteers after ingestion of green tea. Values are geometric means and $95 \% \mathrm{Cl}(n 9)$. 


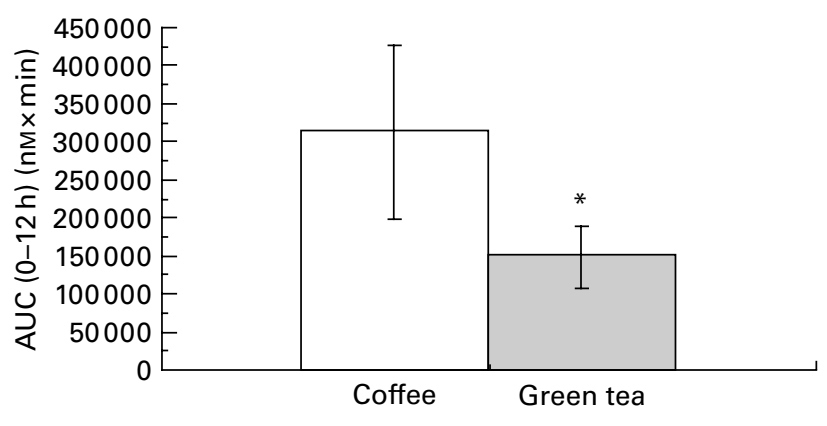

Fig. 3. Comparison of the sum of the area under the curve (AUC) of coffee $(\square)$ and green tea $(\square)$ metabolites $(n 9)$. * Mean values were significantly different $(P=0.0014)$.

\section{Correlations between coffee and tea metabolites}

Based on AUC, correlations between different hydroxycinnamate metabolites present in coffee were highly significant (Table 2). For tea, only EC and EGC were significantly correlated. When the correlations were based on $C_{\max }$ values, only $\mathrm{CA} / \mathrm{iFA}$ and EC/EGC showed a statistical significance (Table 2). Correlation between AUC and $C_{\max }$ was significant for CA, iFA, FA and EGCg (Table 3).

Correlations could not be accurately established for DHFA and DHCA, as return to baseline was not observed in most of the subjects even $12 \mathrm{~h}$ after ingestion. Hence, only truncated AUC was measured for DHCA and DHCA, and $C_{\max }$ could not be determined for all subjects.

\section{Discussion}

Only a few reports on the bioavailability of coffee bioactives have been published ${ }^{(7-11)}$. Coffee contains a complex profile of different isomers of the chlorogenic acids such as caffeoylquinic acid, feruloylquinic acid and di-caffeoylquinic acid, which upon absorption and metabolism will lead to an even

Table 2. Correlation of coffee and tea metabolites based on area under the curve $(\mathrm{AUC})$ and maximum plasma concentration $\left(C_{\max }\right)$

\begin{tabular}{llll}
\hline & & \multicolumn{2}{c}{ Regression $R$} \\
\cline { 3 - 4 } & & AUC & $C_{\text {max }}$ \\
\hline CA & FA & $0.88^{*}$ & 0.36 \\
CA & iFA & $0.91^{*}$ & $0.92^{*}$ \\
FA & iFA & $0.97^{*}$ & 0.60 \\
EC & EGC & $0.69^{*}$ & $0.68^{*}$ \\
EC & EGCg & 0.37 & 0.65 \\
EGC & EGCg & 0.00 & 0.24 \\
CA & EC & 0.26 & 0.05 \\
CA & EGC & 0.23 & 0.01 \\
CA & EGCg & 0.09 & 0.04 \\
FA & EC & 0.26 & 0.10 \\
FA & EGC & 0.00 & 0.18 \\
FA & EGCg & 0.13 & 0.30 \\
iFA & EC & 0.20 & 0.04 \\
iFA & EGC & 0.06 & 0.02 \\
iFA & EGCg & 0.17 & 0.09 \\
\hline
\end{tabular}

CA, caffeic acid; FA, ferulic acid; iFA, isoferulic acid; EC, (-)-epicatechin; EGC, (-)-epigallocatechin; ECg, (-)-epicatechin gallate.

${ }^{*}$ Regression significant at $P<0.05$.
Table 3. Correlation between area under the curve (AUC) and maximum plasma concentration $\left(C_{\max }\right)$ for coffee and tea metabolites

\begin{tabular}{lc}
\hline & $R\left(\mathrm{AUC} / C_{\text {max }}\right)$ \\
\hline CA & $0 \cdot 81^{\star}$ \\
FA & $0 \cdot 62^{\star}$ \\
iFA & $0 \cdot 76^{\star}$ \\
EC & $0 \cdot 48$ \\
EGC & $0 \cdot 17$ \\
EGCg & $0.89^{\star}$ \\
\hline
\end{tabular}

$\mathrm{CA}$, caffeic acid; FA, ferulic acid; iFA, isoferulic acid; EC, (-)epicatechin; EGC, (-)-epigallocatechin; EGCg, (-)-epicatechin gallate.

${ }^{*}$ Regression significant at $P<0.05$.

greater number of metabolites (conjugation and cleavage of the ester bond into phenolic acids). Because of this complexity and to make an attempt to compare with green tea, we narrowed the number of metabolites of interest by cleaving all the molecules of interest into simple phenolic acids, which were measured as equivalents of CA, FA, iFA, DHFA and DHCA as reported previously ${ }^{(11,22)}$. For green tea, several studies have investigated plasma appearance of catechins (EGC, EC and EGCg) $^{(13,14,16,20,21)}$. These data are in agreement with the data reported here on green tea catechins, and show that EGC is the major flavanol appearing in the plasma, and that EGCg is not as well absorbed. In the present study, $C_{\max }$ of EGC peaked at approximately $500 \mathrm{nM}$, while that of EGCg peaked only at approximately $80 \mathrm{nM}$, which is consistent with the published data. Any variation in AUC, $T_{\max }$ and $C_{\max }$ observed in all the studies is dependent on the population studied, the dose and source of green tea. The monophasic phenomenon observed in plasma kinetics of green tea catechins with an early $T_{\max }$ shows that the small intestine is the key site for catechin absorption, and very few colonic metabolites of green tea catechins have been observed in human subjects in vivo ${ }^{(16,23-25)}$. We tried to simplify the analyses using full enzymatic hydrolysis in order to make the overall comparison between coffee and tea and between participants. We are aware that the total number of metabolites for both tea and coffee is very high, and that with improved analytical techniques, the accuracy, detection and quantification of these metabolites will improve as has been highlighted recently for both coffee ${ }^{(10)}$ and tea ${ }^{(25)}$. However, the increased complexity makes the examination of correlations more difficult, since it increases the number of individual compounds.

We further examined the data for differences between the individual volunteers. Based on AUC, we also observed a significant correlation between coffee metabolites. This implies that a subject exhibiting a high AUC for CA would most likely have a high AUC for FA and iFA. Correlation between $\mathrm{CA}$ and iFA was expected, as CA is the precursor of iFA in the hepatic methylation pathway. The correlation between CA and FA was less obvious to predict, as FA may come from either methylation of $\mathrm{CA}$ or from the cleavage of feruloylquinic acid present in the coffee. Inter-individual differences were observed, but individuals with a high level of CA also showed a correspondingly high level of FA ( $r$ 0.88). Similar results were obtained for FA, iFA and CA, 
and also for EGC and EC. However, no such correlation was observed between the tea catechins and coffee phenolic acids. This implies that the mechanisms of absorption for these two classes of compounds are different, and that a high absorber of catechins is not necessarily a high absorber of phenolic acids. For correlations based on $C_{\max }$, the correlation between EC and EGC was also significant, but it was weaker for coffee metabolites and only the correlation between $\mathrm{CA}$ and iFA showed a statistical significance. From the fact that the pathways of catechin metabolism are not as interlinked as those of phenolic acid metabolism, it seems reasonable to predict that correlation coefficients for green tea metabolites would be indeed lower than those for coffee metabolites.

To test the internal consistency for each individual metabolite, we used two parameters, AUC and $C_{\max }$, that were correlated for CA, FA, iFA and EGCg, indicating, as would be expected, that subjects displaying high $C_{\max }$ will most likely have large AUC.

In conclusion, because coffee and green tea are two of the most widely consumed beverages, the present study led us to investigate plasma pharmacokinetics of phenolic acid equivalents and catechins after the consumption of instant coffee and green tea, respectively. Our data show that phenolic acids present in coffee are more bioavailable, on a molar basis, than catechins present in green tea by a factor of 1.7 . This was shown by comparing the sum of the AUC of coffee metabolites to that of the AUC of green tea. This approach remains conservative, as the AUC of coffee metabolites DHCA and DHFA was truncated, and hence was incomplete at $12 \mathrm{~h}$. In the present study, extrapolation of data between 12 and $24 \mathrm{~h}$ for these metabolites was not done to ensure that the data presented were, even though incomplete for DHCA and DHFA, the most reliable to make this coffeegreen tea comparison.

Additionally, the present study confirmed published data on the presence of these bioactives in the plasma after ingestion of the beverages, and based on the measurement of the metabolites for $12 \mathrm{~h}$ after ingestion, we again emphasise the key role of the colon in the metabolism of coffee phenolic acids. Finally, we observed a greater overall correlation between the absorption capacity of the metabolites present in coffee and that of the corresponding catechins present in green tea.

\section{Acknowledgements}

We acknowledge Sylviane Oguey-Araymon, Anny BlondelLubrano, Maurice Beaumont, Bernard Decarli, William Sauret, René Fumeaux and Candice Menozzi-Smarrito for their help and support in the present study. M. R., C. C., H. S. and G. W. designed the research; M. R., C. M. and P. G. conducted the research; D. B. provided essential reagents or materials; M. R., P. G., C. M., A.-L. F. and K. L. analysed the data; J. M. performed statistical analyses; M. R., J. M. and G. W. wrote the paper; M. R. had the primary responsibility for final content; F. D. and G. W. helped with the interpretation of data, discussion and consulting. All authors read and approved the final manuscript. The study reported here was supported in full by Nestec SA. All authors are employees of Nestec SA, which fully funded the present study.

\section{References}

1. Clifford MN (2000) Chlorogenic acids and other cinnamates: nature, occurrence, dietary burden, absorption and metabolism. J Sci Food Agric 80, 1033-1043.

2. Graham HN (1992) Green tea composition, consumption and polyphenol chemistry. Prev Med 21, 334-350.

3. Natella F, Nardini M, Giannetti I, et al. (2002) Coffee drinking influences plasma antioxidant capacity in humans. J Agric Food Chem 50, 6211-6216.

4. Rietveld A \& Wisemann S (2003) Antioxidant effects of tea: evidence from human clinical trials. $J$ Nutr 133, 3285S-3292S.

5. Cabrera C, Artacho R \& Gimenez R (2006) Beneficial effects of green tea - a review. J Am Coll Nutr 25, 79-99.

6. Fukushima Y, Ohie T, Yonekawa Y, et al. (2009) Coffee and green tea as a large source of antioxidant polyphenols in the Japanese population. J Agric Food Chem 57, 1253-1259.

7. Nardini M, Cirillo E, Natella F, et al. (2002) Absorption of phenolic acids in humans after coffee consumption. J Agric Food Chem 50, 5735-5741.

8. Monteiro M, Farah A, Perrone D, et al. (2007) Chlorogenic acid compounds from coffee are differentially absorbed and metabolized in humans. $J$ Nutr 137, 2196-2201.

9. Farah A, Monteiro M, Donangelo CM, et al. (2008) Chlorogenic acids from green coffee extract are highly bioavailable in humans. J Nutr 138, 2309-2315.

10. Stalmach A, Mullen W, Barron D, et al. (2009) Metabolite profiling of hydroxicinnamate derivatives in plasma and urine after the ingestion of coffee by humans: identification of biomarkers of coffee consumption. Drug Metab Dispos 37, $1749-1758$.

11. Renouf M, Guy PA, Marmet C, et al. (2010) Plasma appearance of phenolic acids after ingestion of coffee in humans. Mol Nutr Food Res 54, 1-7.

12. Lee MJ, Wang ZY, Li H, et al. (1995) Analysis of plasma and urinary tea polyphenols in human subjects. Cancer Epidemiol Biomarkers Prev 4, 393-399.

13. Yang CS, Chen L, Lee MJ, et al. (1998) Blood and urine levels of tea catechins after ingestion of different amounts of green tea by human volunteers. Cancer Epidemiol Biomarkers Prev 7, 351-354.

14. Chow HHS, Cai Y, Alberts DS, et al. (2001) Phase I pharmacokinetic study of tea polyphenols following single-dose administration of epigallocatechin gallate and polyphenon E1. Cancer Epidemiol Biomarkers Prev 10, 53-58.

15. Van Amelsvoort JMM, van Het Hof KH, Mathot JNJJ, et al. (2001) Plasma concentration of individual tea catechins after a single oral dose in humans. Xenobiotica 31, 891-901.

16. Lee MJ, Maliakal P, Chen L, et al. (2002) Pharmacokinetics of tea catechins after ingestion of green tea and (-)-epigallocatechin-3-gallate by humans: formation of different metabolites and individual variability. Cancer Epidemiol Biomarkers Prev 11, 1025-1032.

17. Chow HHS, Cai Y, Hakim IA, et al. (2003) Pharmacokinetics and safety of green tea polyphenols after multiple-dose administration of epigallocatechin gallate and polyphenon $\mathrm{E}$ in healthy individuals. Clin Cancer Res 9, 3312-3319.

18. Henning SM, Niu Y, Lee NH, et al. (2004) Bioavailability and antioxidant activity of tea flavanols after consumption of green tea, black tea, or a green tea extract supplement. Am J Clin Nutr 80, $1558-1564$.

19. Chow HHS, Hakim IA, Vining DR, et al. (2005) Effects of dosing condition on the oral bioavailability of green tea catechins after single-dose administration of polyphenon $\mathrm{E}$ in healthy individuals. Clin Cancer Res 11, 4627-4633. 
20. Henning SM, Niu Y, Liu Y, et al. (2005) Bioavailability and antioxidant effect of epigallocatechin gallate administered in purified form versus as green tea extract in healthy individuals. J Nutr Biochem 16, 610-616.

21. Stalmach A, Troufflard S, Serafini M, et al. (2009) Absorption, metabolism and excretion of Choladi green tea flavan-3-ols by humans. Mol Nutr Food Res 53, S44-S53.

22. Guy PA, Renouf M, Barron D, et al. (2009) Guy quantitative analysis of plasma caffeic and ferulic acid equivalents by liquid chromatography tandem mass spectrometry. J Chromatogr B 877, 3965-3974.
23. Mulder TP, Rietveld AG \& van Amelsvoort JM (2005) Consumption of both black tea and green tea results in an increase in the excretion of hippuric acid into urine. Am J Clin Nutr 81, Suppl. 1, 256S-260S.

24. Roowi S, Stalmach A, Mullen W, et al. (2010) Green tea flavan-3-ols: colonic degradation and urinary excretion of catabolites by humans. J Agric Food Chem 58, 1296-1304.

25. Del Rio D, Calani L \& Cordero C, et al. (2010) Bioavailability and catabolism of green tea flavan-3-ols in humans. Nutrition (Epublication ahead of print version 14 January 2010). 